\title{
CONTROLE E MONITORAMENTO NO TRIATHLON: ESTUDO A PARTIR DA PERCEPÇÃO SUBJETIVA DO ATLETA NO PERÍODO PRÉ-COMPETITIVO
}

Gerson dos Santos Leite

João Paulo Borin

Guilherme Souza Lobo Moreira Salles

Vitor Carrara

Orival Andries Junior

\section{Resumo}

A busca por indicadores das respostas imediatas obtidas durante as sessões de treinamento tem sido alvo de investigações no âmbito científico atual, neste sentido o objetivo do estudo foi verificar correlação entre indicadores da carga de treinamento de um triatleta no período pré-competitivo. Para análise dos dados utilizou-se medidas descritivas de centralidade e dispersão e o coeficiente (r) de correlação de Pearson $(\mathrm{p}<0,05)$. Os resultados apontam tendência de aumento do volume, da carga e das percepções de cansaço ao longo dos dias de treinamento. Em relação as semanas, verifica-se uma tendência de aumento para a carga, de diminuição da monotonia e manutenção do strain. Pode-se concluir que as variáveis analisadas parecem permitir o monitoramento das alterações no treinamento possibilitando sua caracterização.

\section{Palavras-Chave}

Treinamento desportivo; Triathlon; Preparação.

\section{CONTROL AND MONITORING IN TRIATHLON: STUDY FROM THE ATHLETE'S SUBJECTIVE PERCEPTION IN THE PERIOD PRE-COMPETITIVE}

Gerson dos Santos Leite

João Paulo Borin

Guilherme Souza Lobo Moreira Salles

Vitor Carrara

Orival Andries Junior

\begin{abstract}
The search for indicators of immediate answers obtained during the training sessions has been being target of investigations in the current scientific scope, in this sense the study goal was to verify correlation between training load indicators of a triathlete in the period pre-competitive. For data analysis it used descriptive measures of centralness and dispersion and the coefficient ( $r$ ) of Pearson's correlation $(p<0,05)$. The results point increase tendency of the volume, of the load and of the weariness perceptions along the training days. In relation weeks, it is verified an increase tendency for the load, decrease in monotony and maintenance of strain. It can conclude that the analyzed variables seem to allow monitoring of the alterations in the training enabling her characterization.
\end{abstract}

\section{Key-Words}

Sport training; Triathlon; Preparation. 


\section{INTRODUÇÃO}

O monitoramento fisiológico mostra-se como ferramenta importante no controle das adaptações geradas pelo treinamento (GUEZENNEC et al., 1996; MILLET, et al., 2000; BOUSSANA et al., 2003), mas necessita de equipamentos sofisticado e pessoal especializado, o que eleva o custo operacional de sua prática. A busca por metodologias acessíveis e válidas que possam auxiliar no treinamento de triathlon tem sido o objetivo de recentes pesquisas (COUTTS; SLATTERY; WALLACE, 2007; COUTTS; WALLACE; SLATTERY; 2007; GONZÁLEZ-HARO et al., 2007), que têm como foco o monitoramento do desempenho do atleta (PATON; HOPKINS, 2005) e as variáveis relacionadas à carga de treinamento (VELOSO et al., 2005). No triathlon ainda são escassas as pesquisas que relacionem metodologias de fácil aplicabilidade e o processo de treinamento.

No âmbito do treinamento desportivo é fundamental controlar diferentes variáveis do treinamento que se relacionem com a carga aplicada (BORIN; GOMES; LEITE, 2007) além de analisar os efeitos de diferentes metodologias de treino no estado do atleta (VERKOSHANSK, 1996; MOREIRA et al., 2006).

A percepção subjetiva de esforço (PSE) tem sido utilizada durante trabalhos físicos como ferramenta que aponte informações gerais de trabalho muscular, cardiovascular, função respiratória e do sistema nervoso central (BORG, 1982). Recentemente, Foster (1998) propôs que a intensidade da sessão de treinamento poderia ser avaliada pela percepção subjetiva de esforço e junto ao volume do treino em minutos era possível quantificar a carga de treinamento. Também formulou o conceito de monotonia e strain do treinamento que seriam um o indicador de variação da carga na semana e o outro índice de esforço semanal relacionado à carga e sua variação. Cabe destacar que, quando tais indicadores demonstrassem grande variância em relação á outras semanas os atletas poderiam estar sujeitos a enfermidades e lesões (FOSTER, 1998; PUTLUR et al., 2004; SUZUKI et al., 2006). Seiler e Kjerland (2006) demonstraram que tal método foi capaz de monitorar a intensidade dos treinamentos de atletas fundistas e apresentavam os mesmos resultados quando comparados à freqüência cardíaca e lactato para monitorar a intensidade.

Para conhecer e entender melhor os efeitos do treinamento, alguns autores têm proposto avaliar a carga aplicada durante o macrociclo de treinamento junto as variáveis qualitativas (intensidade) e quantitativa (volume) que a compõem (JONES, 1998; MOREIRA et al., 2004), demonstrando a efetividade da distribuição das cargas ou mesmo a importância de uma reestruturação.

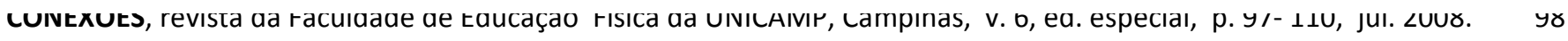
ISSN: $1983-9030$. 
Neste sentido, o objetivo deste trabalho foi verificar se a carga, seus componentes e a percepção subjetiva de cansaço podem ser indicadores para o monitoramento do treinamento de triatlon.

\section{MATERIAIS E MÉTODOS}

A pesquisa caracteriza-se como estudo de caso com acompanhamento transversal, pois buscou-se entender a dinâmica de alteração da carga e percepção de cansaço ao longo de cinco semanas de treinamento (PEREIRA, 1995).

\section{SUJEITO}

Um atleta de triathlon de alto desempenho, envolvido em treinamento sistematizado para a modalidade há 12 anos, participante de provas nacionais e internacionais, com idade de 27 anos, 1,81m de estatura e peso de $72 \mathrm{~kg}$.

\section{PROCEDIMENTOS GERAIS DO CONTROLE E MONITORAMENTO DO TREINAMENTO}

O controle e monitoramento do treinamento do atleta foram realizados durante cinco semanas do período pré-competitivo, totalizando 34 dias de atividades e 69 sessões de treinamento (no período de 15/01 a 18/02/07). O objetivo da organização dos treinamentos centrou-se em buscar o melhor resultado no Campeonato Brasileiro de Triathlon de Longa Distância que possuía como característica quanto à distância: $3 \mathrm{~km}$ de natação, $80 \mathrm{~km}$ de ciclismo e $20 \mathrm{~km}$ de corrida. Cabe aqui destacar que o referido indivíduo analisado sagrou-se Campeão Brasileiro em sua categoria (25-29 anos) o que lhe garantiu vaga para disputar o Mundial de Triathlon de Longa Distância disputado em Lorient - França em julho do mesmo ano.

Para um efetivo controle e monitoramento do treinamento toda a coleta de dados foi realizada pelo mesmo avaliador imediatamente antes e após as sessões de treinamento, buscando assim confiabilidade nos dados coletados (PEREIRA; OMES, 2003).

Durante o período de treinamento analisado, o atleta foi submetido à avaliação de caráter formativo, que tem como característica a coleta de informações diariamente durante as sessões de treino (MARINS;GIANNICHI, 2003).

\section{AVALIAÇÃO dO CANSAÇO DIÁRIO dO ATLETA}

A percepção subjetiva de cansaço do atleta foi avaliada baseada na escala de esforço proposta por Borg CONEXÕES, revista da Faculdade de Educação Física da UNICAMP, Campinas, v. 6, ed. especial, p. 97- 110, jul. 2008. ISSN: $1983-9030$. 
(2000) com adaptação de Jurado e Borin (2006). Coletada diariamente antes e após cada sessão de treinamento (tabela 1), o avaliador se referia ao atleta nos momentos descritos da seguinte forma: "Qual seu estado de cansaço neste momento de acordo com a escala? ".

Tabela 1 - Escala de Cansaço com 15 níveis (6-20) (JURADO, BORIN, 2006).

\begin{tabular}{|c|c}
\hline & Escala de Cansaço \\
6 & \\
7 & Muito, Muito Bem \\
8 & \\
9 & Muito Bem \\
10 & Bem \\
11 & \\
12 & Pouco Cansado \\
13 & \\
14 & Cansado \\
15 & Muito Cansado \\
16 & \\
17 & \\
18 & Muito, Muito Cansado \\
19 & Exausto \\
20 & \\
\hline
\end{tabular}

\section{MONITORAMENTO DO VOLUME, DA INTENSIDADE E DA CARGA DE TREINAMENTO.}

Diariamente após cada sessão de treinamento anotou-se a quantidade de treinamento da sessão (em quilômetros e em minutos). Associado a este procedimento, era apresentada ao atleta a escala de percepção subjetiva de intensidade (PSI) proposta por Foster (1998) para calcular a carga de cada sessão (tabela 2). O avaliador dirigia-se ao atleta e formulava a seguinte pergunta: "Qual foi a intensidade do treinamento em relação à escala?". Ao final de cada microciclo foi determinada a carga média da semana e sua variação (desvio padrão), utilizados para o cálculo da monotonia (indicador de variação semanal), índice este que, junto à carga total semanal (soma das diárias) foi utilizado para o cálculo do strain (índice de estresse aplicado ao atleta na semana), pelas seguintes fórmulas (FOSTER, 1998):

Carga diária = PSI x duração da sessão diária;

Carga semanal total $=\Sigma$ Carga diária;

Carga semanal média $=\Sigma$ carga diária $/ \Sigma$ dias de treinamento;

Monotonia = carga semanal média / desvio padrão das cargas diárias;

Strain $=$ Monotonia $\mathrm{x}$ carga semanal total. 
Tabela 2 - Escala de intensidade do treinamento (FOSTER, 1998).

\section{Escala de Intensidade}

\begin{tabular}{cc}
\cline { 2 - 2 } 0 & Repouso \\
\hline 1 & Muito Muito \\
2 & Fraco \\
3 & Moderado \\
4 & Algo Forte \\
5 & Forte \\
6 & \\
7 & Muito Forte \\
8 & \\
9 & \\
10 & Máximo \\
\hline
\end{tabular}

Cabe aqui destacar que a carga e suas variações são representadas por meio de unidades arbitrárias (u.a.).

\section{MÉTODO ESTATÍSTICO}

Os dados coletados foram transferidos para banco computacional e produziram-se informações no plano descritivo, por meio de medidas de centralidade e dispersão. Para verificar possíveis relações entre as varáveis analisadas foi utilizado o coeficiente ( $r$ ) de correlação de Pearson, com $p<0,05$ (NORMAN e STREINER, 1994). Na tentativa de obter uma projeção dos dados utilizou a reta de tendência linear considerando o conjunto de dados.

\section{RESULTADOS}

Após a coleta dos dados, produziu-se informação com a característica geral do monitoramento do treinamento (tabela 3) e a variação diária e semanal das variáveis analisadas (gráficos 1 ao 6). Por fim a tabela 4 aponta para resultado da correlação entre as variáveis estudadas. 
Tabela 3 - Variáveis monitoradas durante as 5 semanas de treinamento quanto a carga, cansaço, volume semanal e quilometragem percorrida nas modalidades.

\begin{tabular}{|c|c|c|c|c|c|c|}
\hline \multirow{2}{*}{\multicolumn{2}{|c|}{ Variável estudada }} & \multicolumn{5}{|c|}{ Semanas } \\
\hline & & 1 & 2 & 3 & 4 & 5 \\
\hline \multirow{5}{*}{ Carga (u.a.) } & Semanal & 4033 & 4313 & 3444 & 4727 & 5158 \\
\hline & Média & 576,1 & 616,1 & 492 & 675,3 & 736,9 \\
\hline & Desvio Padrão & 279,0 & 217,7 & 255,3 & 343,7 & 375,6 \\
\hline & Monotonia & 2,1 & 2,8 & 1,9 & 1,9 & 2,0 \\
\hline & Strain & 8330 & 12205 & 6638 & 9287 & 10120 \\
\hline \multirow{3}{*}{$\begin{array}{l}\text { Percepção de } \\
\text { Cansaço (u.a.) }\end{array}$} & Inicial & 10,6 & 11,8 & 11,4 & 11,4 & 12,8 \\
\hline & Final & 12,4 & 13,9 & 13,0 & 13,1 & 14,3 \\
\hline & $\begin{array}{c}\text { PSI } \\
\text { Tempo }\end{array}$ & 3,6 & 4,0 & 3,8 & 3,8 & 4,1 \\
\hline \multirow[t]{2}{*}{ Volume } & $\begin{array}{c}\text { Semanal (min) } \\
\text { Percurso }\end{array}$ & 1085 & 1102 & 936 & 1278 & 2286 \\
\hline & Semanal $(\mathrm{km})$ & 329 & 354 & 303 & 393 & 353 \\
\hline \multirow{3}{*}{$\begin{array}{l}\text { Modalidades } \\
(\mathrm{km})\end{array}$} & Natação & 10 & 12 & 8 & 13 & 15 \\
\hline & Ciclismo & 270 & 290 & 250 & 320 & 270 \\
\hline & Comida & 49 & 52 & 45 & 60 & 68 \\
\hline
\end{tabular}

As Figuras 1 e 2 apontam para o comportamento dos resultados do monitoramento diário e semanal das variáveis analisadas no presente estudo. Destaca-se na primeira, a tendência de crescimento do volume em minutos (1A) e da carga de treinamento (1C) ao longo dos dias, juntamente com o aumento da percepção de cansaço do atleta (1D), tanto inicial como final, apesar da intensidade referida apresentar estabilidade ao longo das semanas analisadas (1B). Na figura 2, destaca-se a tendência de aumento da carga semanal (2A) e de diminuição da monotonia (2B), além de estabilização ao analisar o strain $(2 \mathrm{C})$. 
Figura 1- Volume diário dos treinamentos em minutos (A), intensidade diária relativa a escala de Foster (1998) (B), carga diária dos treinamentos (C) e cansaço inicial e final de treinamento diário (D) e suas respectivas linhas de tendência.
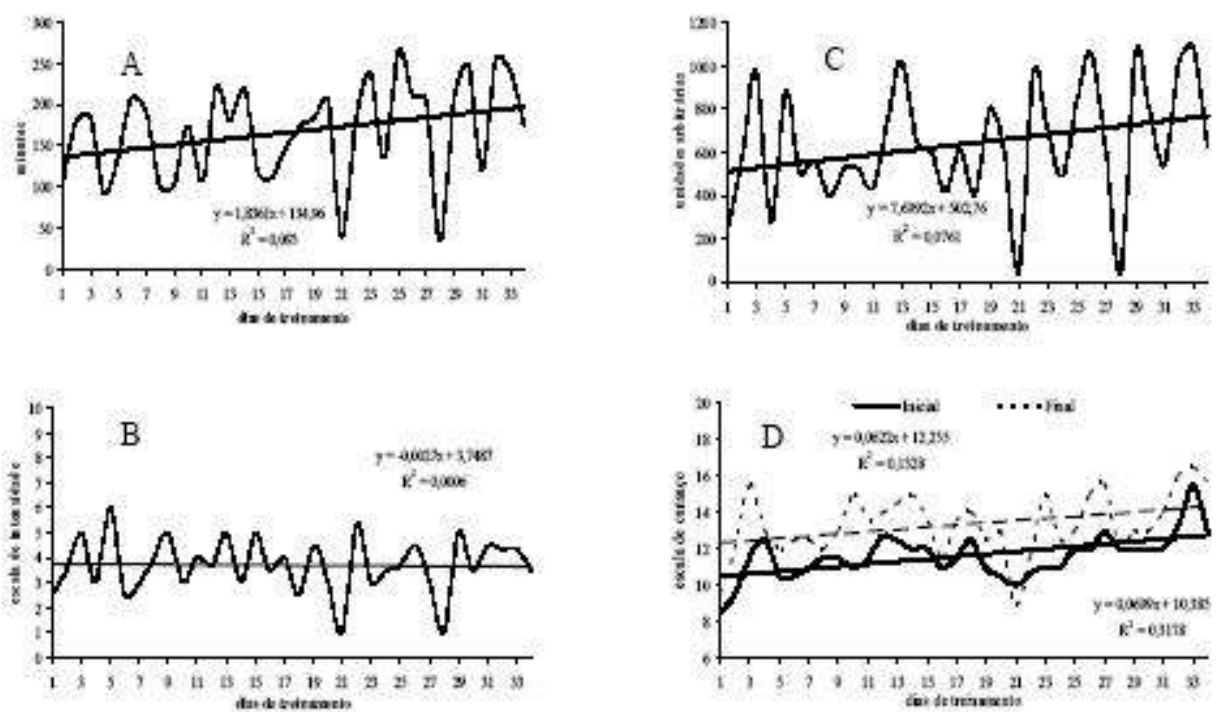

Figura 2- Carga semanal de treinamento (A), monotonia (B), Strain (C), e suas respectivas linhas de tendência
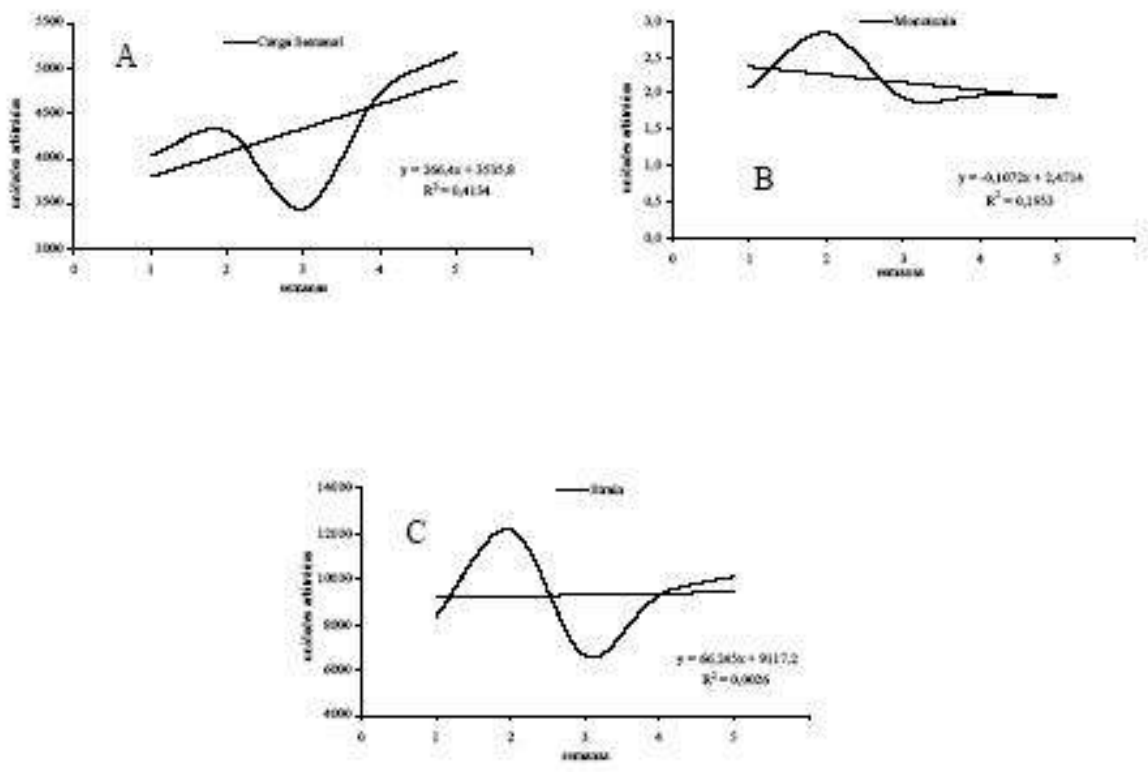


\section{Tabela 4- Distribuição dos valores de correlação entre variáveis analisadas}

\begin{tabular}{|c|c|c|c|c|c|c|c|c|}
\hline Variàveis & $\mathrm{CS}$ & $\mathrm{CM}$ & Mon & Strain & $\mathrm{CI}$ & $C F$ & Intensidade & VS $(\mathrm{km})$ \\
\hline $\mathrm{CM}$ & 1,00 & & & & & & & \\
\hline Monotonia & $-0,02$ & $-0,02$ & & & & & & \\
\hline Strain & 0,60 & 0,60 & 0,79 & & & & & \\
\hline PSCI & 0,65 & 0,65 & 0,06 & 0,46 & & & & \\
\hline PSCF & 0,65 & 0,65 & 0,33 & 0,67 & $0,96^{*}$ & & & \\
\hline Intensidade & 0,64 & 0,64 & 0,42 & 0,74 & $0,93 *$ & $0,99 *$ & & \\
\hline VS (km) & 0,77 & 0,77 & 0,13 & 0,58 & 0,23 & 0,30 & 0,32 & \\
\hline VS (min) & 0,84 & 0,84 & $-0,26$ & 0,31 & 0,82 & 0,72 & 0,68 & 0,32 \\
\hline
\end{tabular}

\section{DISCUSSÃO}

No âmbito desportivo, a busca por indicadores que aponte os resultados mais próximos da realidade do treinamento tem sido alvo de investigação. Particularmente, ao observar os dados do presente trabalho, verifica-se questões interessantes a serem discutidos.

A primeira volta-se a utilização de metodologia que considere diferentes aspectos que envolvem o atleta. De fato, ao verificar a determinação do cansaço tanto inicial quanto final de treinamento a princípio parece ter sido influenciado pela carga, pois houve uma tendência de aumento durante o período analisado. Tais dados corroboram com os resultados apresentados por Leite (2007), que encontrou correlação significativa entre carga total da semana e o cansaço inicial e final de treinamento $(r=0,29$ e $r$ $=0,30$ com $\mathrm{p}<0,005$ e $\mathrm{p}<0,001$ respectivamente). A tabela 4 mostra que a percepção de cansaço do atleta, seja antes ou depois do treino foi influenciada pela intensidade do treinamento, dada pela correlação encontrada ( $r=0,93$ e $r=0,99$; com $p<0,05$ respectivamente). Tais resultados demonstram que nas semanas analisadas, quanto maior foi à intensidade empregada no treino, maior seria o estado de cansaço do atleta após este treino e no início do próximo, confirmada pela correlação encontrada $(r=0,96$ com $\mathrm{p}<0,05)$ entre cansaço final e inicial. A percepção de cansaço do triatleta parece ser um indicador da relação estímulo-pausa durante os treinos podendo ser utilizada como forma de controle do treinamento, pois este permite conhecer o estado atual do atleta e corrigir programas de treinamento quando necessário (BORIN; GOMES; LEITE, 2007).

O método proposto por Foster (1998) parece ser adequado para o monitoramento e controle do

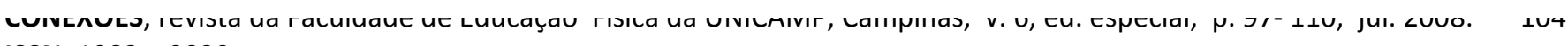
ISSN: $1983-9030$. 
treinamento de triathlon, já que demonstrou diferentes características das semanas analisadas conforme apresentado na tabela 3 e figura 1 e 2 . A carga de treinamento indica tendência de aumento, contrária a monotonia que apresenta decréscimo ao longo das semanas e ao strain que mesmo com uma variação remete a estabilização.

Outro ponto a considerar, volta-se à quantificação da carga mostra-se necessário para a caracterização das sessões de treinamento e o acompanhamento de sua distribuição nas diferentes semanas de trabalho, aqui representada pela monotonia, que representa a variação desta carga na semana (FOSTER et al., 2005). Valor alto em seu score está relacionado a condições desfavoráveis para ótima adaptação ao treinamento (FOSTER, 1998; SUZUKI et al., 2006). Foster et al. (2005) descrevem que valores de monotonia acima de 2 unidades arbitrárias (u.a.) contribuem para o desenvolvimento da síndrome de overtraining.

O monitoramento ao longo das semanas permite indicar que durante a segunda semana de treinamento as cargas de treinamento não foram bem distribuídas, já que a monotonia teve seu valor elevado $(2,8$ u.a.). Este comportamento da monotonia aponta que as cargas de treinamento poderiam ser distribuídas diferentemente nas semanas. Fry, Morton, Keast (1992) descrevem a importância da adequada distribuição das cargas de treinamento, com suficientes períodos de recuperação para melhorar o desempenho dos atletas, além de prevenir o overtraining.

O valor do Strain da semana, entendido aqui como o produto da multiplicação da carga semanal pela monotonia é um índice que reflete o "esforço" da semana, pois é o produto duma inter-relação do que foi aplicado (carga) com sua variação semanal (monotonia). Apesar do strain não apresentar na literatura um score ideal ou mesmo limitador que indique o momento de se trabalhar com uma semana regenerativa posterior a uma com strain elevado, tendo como base a semana ou as semanas anteriores, já que ele tem sido relacionado com os sintomas de overtraining, mas especificamente com as ocorrências de infecções banais do trato respiratório superior (FOSTER, 1998) e com a alteração da função imune em atletas (PUTLUR et al., 2004). No treinamento analisado parece ter sido planejado uma semana regenerativa (terceira) após uma com alto strain (segunda), pois a terceira semana teve uma queda de $20 \%$ na carga semanal e de $45,6 \%$ no strain comparada a segunda.

Alguns autores (DELLATRE et al. 2006; FOSTER, 1998; LEITE, 2007; PUTLUR et al., 2004) verificaram que a variação da carga na semana (monotonia) é o que parece mais influenciar o strain,

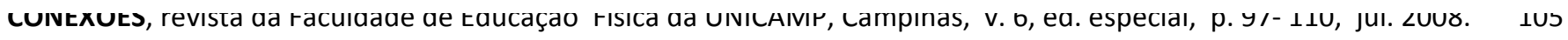
ISSN: $1983-9030$. 
pois um alto valor de monotonia estaria relacionado com um alto strain, demonstrando a importância de uma adequada distribuição da carga de treinamento. Mesmo a segunda semana não ter sido a com o maior volume ou intensidade de treinamento, pode ser classificada como a mais "estressante" para o atleta, devido ao alto valor de strain e monotonia encontrado.

O controle formativo do strain, além de demonstrar sua variação ao longo do tempo, fornece parâmetros para o controle da solicitação do atleta durante os treinamentos e, principalmente, pela fácil aplicabilidade do método, mostra-se bastante confiável para avaliar a relação entre a duração (volume) e a intensidade da sessão de treino, não requerendo uso de equipamentos tecnológicos com altos custos e refletindo indicadores fisiológicos, como a freqüência cardíaca e lactato sanguíneo (FOSTER et al., 2001; SEILER;KJERLAND, 2006).

\section{CONCLUSÃO}

A partir dos resultados obtidos, conclui-se que:

- A percepção subjetiva de cansaço parece ser indicador real da relação estímulo-pausa no treinamento analisado;

- A escala proposta por Foster (1998) foi capaz de monitorar as alterações na intensidade de treinamento o que permitiu a caracterização do treino;

- A monotonia e o strain parecem ser indicadores importantes para serem utilizados no controle e monitoramento do treinamento.

Por fim, ressalta-se a necessidade de novos estudos com o objetivo de avaliar e relacionar diferentes variáveis presentes no treinamento e que apontem para respostas no auxílio tanto no planejamento como no controle e monitoramento dos efeitos de treinamento.

Agradecimento especial para a Coordenação de Aperfeiçoamento de Pessoal de Ensino Superior CAPES pela concessão de bolsa no período de coleta dos dados. 


\section{REFERÊNCIAS}

BORG, G. Escala de Borg para dor e o esforço percebido, São Paulo: Manole, 2000.

. Psychophysical bases of perceived exertion. Medicine and Science in Sports and Exercise, v. 14, n. 5, p. 377-381, 1982.

BOUSSANA, A. et al. The effects of prior cycling and a successive run on respiratory muscle performance in triathletes. International Journal of Sports Medicine, p. 63-70, 2003.

COUTTS, A. J.; SLATTERY, K. M.; WALlACE, L. K. Practical tests for monitoring performance, fatigue and recovery in triathletes. Journal of Science and Medicine in Sport, 2007. (in press).

COUTTS, A. J.; WAllaCE, L. K.; SlATTERY, K. M. Monitoring changes in performance, physiology, biochemistry, and psychology during overreaching and recovery in triathletes. International Journal of Sports Medicine, v. 28, p. 125-134, 2007.

DELATTRE, E.; GARCIN, M.; MILLE-HAMARD, L.; BILLAT, V. Objective and subjective analysis of the training content in young cyclists. Applied Physiology Nutricion Metabolism, v. 31, p.118-125, 2006.

FOSTER, C. Monitoring training in athletes with reference to overtraining syndrome. Medicine and Science in Sports and Exercise, v. 30, n. 7, p.1164-1168, 1998.

FOSTER, C. et al. New approach to monitoring exercise training. Journal of Strenght and Condittioning Research, v.15, n.1, p.109-115, 2001.

FOSTER, C; HOYOS, J.; LUCIA, A. Regulation of energy expenditure during prolonged athletic competition. Medicine and Science in Sports and Exercise, v. 37, n. 4, p. 670-675, 2005.

FRY, R. W.; MORTON A. R.; KEAST D. Periodisation and the prevention of overtraining. Canadian Journal Sports Science, v. 17, n. 3, p. 241-248, 1992.

GONZÁLEZ-HARO, C. et al. Validation of a field test to determine the maximal aerobic power in triathletes and endurance cyclists. British Journal of Sports Medicine, v. 41, p. 174-179, 2007.

GUEZENNEC, C. Y. et al. Increase in energy cost of running at the end of a triathlon. Journal Applied Physiology, v. 73, p. 440-445, 1996.

JONES, A. M. A five year physiological case study of an olympic runner. British Journal of Sports Medicine, v. 32, n. 1, p. 39-43, 1998.

JURADO, S.; BORIN, J. P. Avaliação dos efeitos neuromusculares do treinamento de força em atletas de basquetebol. In: MOSTRA ACADÊMICA DA UNIMEP, 4. 2006, Piracicaba. Anais... Piracicaba:UNIMEP, 2006.

CONEXÕES, revista da Faculdade de Educação Física da UNICAMP, Campinas, v. 6, ed. especial, p. 97- 110, jul. 2008. 107 ISSN: $1983-9030$. 
LEITE, G. S; et al. Limiar anaeróbio indireto prediz desempenho no short triathlon. Saúde em Revista, v. 9, n. 21, p.7-12, 2007.

MARINS, J. C. B.; GIANNICHI, R. S. Avaliação e prescrição de atividade física: guia prático. Rio de Janeiro: Shape, 2003.

MILlet, G. P., VLECK, V. E. Physiological and biomechanical adaptations to the cycle to run transition in olympic triathlon: review and practical recommendations for training. British Journal of Sports Medicine, v. 34, p. 384-390, 2000.

MOREIRA A. A eficácia e a heterocronia das respostas de adaptação de basquetebolistas submetidos a diferentes modelos de estruturação. 2006. Tese (Doutorado) - Faculdade de Educação Física, Universidade Estadual de Campinas, Campinas, 2006.

MOREIRA, A. et al. A dinâmica de alteração das medidas de força e o efeito posterior duradouro de treinamento em basquetebolistas submetidos ao sistema de treinamento em bloco. Revista Brasileira de Medicina do Esporte, v. 10, n. 4, p. 243-250, 2004.

NORMAN, G. R.; STREINER, D. L. Biostatistics: the bare essentials. Mosb Year Book: St. Louis, 1994.

PATON, I. C. D.; HOPKINS, W.G. Seasonal changes in power of competitive cyclists: implications for monitoring performance. Journal Science and Medicine Sport, v. 8, n. 4, p. 375-381, 2005.

PEREIRA, M. G. Epidemiologia: teoria e prática. Rio de Janeiro: Guanabara Koogan, 1995.

PEREIRA, M. I. R.; GOMES, P. S. C. Testes de força e resistência muscular: confiabilidade e predição de uma repetição máxima, revisão e novas evidências. Revista Brasileira de Medicina do Esporte, v. 9, n. 5, p. 325-335, 2003.

PLUTUR, P. et al. Alteration of immune function in womem collegiate soccer players and college students. Journal or Sports Sciences and Medicine, v. 3, p. 234-243, 2004.

SEILER, K. S.; KJERLAND G. O., Quantifying training intensity distribution in elite endurance athletes: is there evidence for an "optimal" distribution? Scandinavian Journal of Medicine and Science in Sports, v. 6, p. 49-56, 2006.

SUZUKI, S. et al. Program design basead on a mathematical model using rating of perceived exertion for an elite japanese sprinter: a case study. Journal of Strength and Conditioning Research, v. 20, n.1, p. 36-42, 2006.

VELOSO, J. H. S. et al. Controle da carga de treinamento no triathlon. In: SIMPÓSIO INTERNACIONAL DE INICIAÇÃO CIENTÍFICA, 13., 2005, Ribeirão Preto. Anais... Ribeirão

CONEXÕES, revista da Faculdade de Educação Física da UNICAMP, Campinas, v. 6, ed. especial, p. 97- 110, jul. 2008.108 ISSN: $1983-9030$. 
Preto:USP, 2005.

VERKHOSANSKY, Y. V. Força: treinamento da potência muscular. Londrina: Centro de Informações Desportivas, 1996. 


\section{Gerson dos Santos Leite}

Faculdade de Educação Física - UNIMEP - UNINOVE/São Paulo

E-mail: gersonslt@gmail.com

\section{João Paulo Borin}

Faculdade de Educação Física- UNIMEP

\section{Guilherme Souza Lobo Moreira Salles}

Faculdade de Educação Física- UNIMEP

\section{Vitor Carrara}

Equipe Ironcoach de Triathlon

\section{Orival Andries Junior}

Faculdade de Educação Física - Unicamp

\section{Referência do artigo:}

\section{ABNT}

LEITE, G. S., et al, Controle e monitoramento no triathlon: estudo a partir da percepção subjetiva do atleta no período pré-competitivo. Conexões, v.6, ed. especial, p. 97-110, 2008

\section{APA}

Leite, G. S., Borin, J. P., Salles, G. S. L. M., Carrara, V., \& Andries Junior, O. (2008). Controle e monitoramento no triathlon: estudo a partir da percepção subjetiva do atleta no período précompetitivo. Conexões, 6(ed. especial), 97-110.

\section{VANCOUVER}

Leite GS, Borin J.P, Salles GSLM, Carrara V, Andries Junior O. Controle e monitoramento no triathlon: estudo a partir da percepção subjetiva do atleta no período pré-competitivo. Conexões, 2008; 6(ed. especial): 97-110. 